\title{
In vitro and in vivo evaluation of electrochemotherapy with trans-platinum analogue trans-[ $\left.\mathrm{PtCl}_{2}(3-\mathrm{Hmpy})_{2}\right]$
}

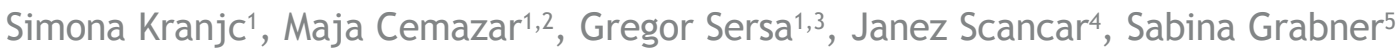 \\ ${ }^{1}$ Institute of Oncology Ljubljana, Department of Experimental Oncology, Ljubljana, Slovenia \\ 2 University of Primorska, Faculty of Health Sciences, Izola, Slovenia \\ ${ }^{3}$ Faculty of Health Sciences, University of Ljubljana, Ljubljana, Slovenia \\ ${ }^{4}$ Department of Environmental Sciences, Jozef Stefan Institute, Ljubljana, Slovenia \\ ${ }^{5}$ Faculty of Chemistry and Chemical Technology, University of Ljubljana, Ljubljana, Slovenia
}

Radiol Oncol 2017; 51(3): 295-306.

Received 20 July 2017

Accepted 4 August 2017

Correspondence to: Simona Kranjc, Ph.D., Department of Experimental Oncology, Institute of Oncology Ljubljana, Zaloška 2, SI-1000 Ljubljana, Slovenia. Phone/Fax: +386 15879 434; E-mail: skranjc@onko-i.si

Disclosure: No potential conflicts of interest were disclosed.

Background. Cisplatin is used in cancer therapy, but its side effects and acquired resistance to cisplatin have led to the synthesis and evaluation of new platinum compounds. Recently, the synthesized platinum compound trans$\left[\mathrm{PtCl}_{2}(3-\mathrm{Hmpy})_{2}\right]$ (3-Hmpy = 3-hydroxymethylpyridine) (compound 2) showed a considerable cytotoxic and antitumour effectiveness. To improve compound $\mathbf{2}$ cytotoxicity in vitro and antitumour effectiveness in vivo, electroporation was used as drug delivery approach to increase membrane permeability (electrochemotherapy).

Materials and methods. In vitro, survival of sarcoma cells with different intrinsic sensitivity to cisplatin (TBLCl2 sensitive, TBLCI2Pt resistant and SA-1 moderately sensitive) was determined using a clonogenic assay after treatment with compound $\mathbf{2}$ or cisplatin electrochemotherapy. In vivo, the antitumour effectiveness of electrochemotherapy with compound $\mathbf{2}$ or cisplatin was evaluated using a tumour growth delay assay. In addition, platinum in the serum, tumours and platinum bound to the DNA in the cells were performed using inductively coupled plasma mass spectrometry.

Results. In vitro, cell survival after treatment with compound $\mathbf{2}$ electrochemotherapy was significantly decreased in all tested sarcoma cells with different intrinsic sensitivity to cisplatin (TBLCl2 sensitive, TBLCI2Pt resistant and SA-1 moderately sensitive). However, this effect was less pronounced compared to cisplatin. Interestingly, the enhancement factor (5-fold) of compound $\mathbf{2}$ cytotoxicity was equal in cisplatin-sensitive TBLCl2 and cisplatin-resistant TBLCI2Pt cells. In vivo, the growth delay of subcutaneous tumours after treatment with compound $\mathbf{2}$ electrochemotherapy was lower compared to cisplatin. The highest antitumour effectiveness after cisplatin or compound 2 electrochemotherapy was obtained in TBLCl2 tumours, resulting in $67 \%$ and $11 \%$ of tumour cures, respectively. Compound $\mathbf{2}$ induced significantly smaller loss of animal body weight compared to cisplatin. Furthermore, platinum amounts in tumours after compound $\mathbf{2}$ or cisplatin electrochemotherapy were approximately 2-fold higher compared to the drug treatment only, and the same increase of platinum bound to DNA was observed.

Conclusions. The obtained results in vitro and in vivo suggest compound $\mathbf{2}$ as a potential antitumour agent in electrochemotherapy.

Key words: platinum analogue; cisplatin; 3-Hmpy; electroporation; electrochemotherapy; mouse sarcoma

\section{Introduction}

Cisplatin is used for the treatment of variable types of cancers (testicular, bladder, ovarian, en- dometrial, cervical, lung, head and neck), and primarily systemically used as single drug or in combination with other drugs and/or in combination with radiation therapy, immunotherapy or 
other targeted therapies. ${ }^{1-3}$ Despite many efforts in adapting an appropriate therapy schedule with cisplatin, the treatment of patients with cisplatin induces side effects, and cancer cells can acquire resistance to cisplatin through different mechanisms. Among these mechanisms, the inactivation of cisplatin via thiol-containing molecules, such as glutathione and metallothionein, increased the repair of cisplatin-DNA adducts, enhanced tolerance to cisplatin-DNA adducts, resulting in the failure of apoptotic pathways and reduction of platinum accumulation through decreased drug uptake or increased drug efflux. ${ }^{4-8}$ Therefore, overcoming side effects and acquired resistance of cells to cisplatin during the treatment of patients with cisplatin still remains the main challenge in studies of platinum analogues. Modification of platinum compounds with variable ligands (iminoethers, aliphatic amines, amine piperazine and pyridine) in the trans position of ligands resulted in variable cytotoxic effects. ${ }^{9-17}$ Pyridine ligands with hydroxyl groups, which participate in the formation of stable complex between DNA and platinum compounds, were demonstrated as promising ligands in activating platinum (II) compound cytotoxicity. $9,11,14,18-20$ Recently, we reported that two 3-hydroxymethylpyridine (3-Hmpy) ligand in the trans position in the platinum (II) complex increased cytotoxicity in vitro and antitumour effectiveness in vivo. ${ }^{21}$

The effectiveness of chemotherapeutic drugs relies on their uptake into tumour cells. For poorly permeable drugs, higher doses are needed for effective cytotoxicity to tumour cells, consequently producing higher side effects. Cisplatin is a poorly permeable drug; therefore, many approaches have been examined to increase its uptake, i.e., using nanoparticles, magnetic particles, liposomes, and tumour-specific antibodies and chemically or physically inducing pores in the cell membrane. ${ }^{16,22-23}$ Among physical approaches, electroporation, inducing pores in the cell membrane via electric pulses, could be used. ${ }^{24}$ Electroporation increases the cytotoxicity up to 80-fold in cisplatin-sensitive and cisplatin-resistant tumour models, in vitro and in vivo. ${ }^{25-29}$ The treatment of tumour nodules with the local application of electric pulses after systemic or local administration of drug is referred to as electrochemotherapy. Electrochemotherapy with cisplatin is currently used for the treatment of variable cutaneous and subcutaneous tumours in human and veterinary clinics, showing a local effectiveness of up to $80 \%$ of local tumour control. ${ }^{30-33}$ The antitumour effectiveness of electrochemotherapy primarily relies on increased drug uptake and platinum binding to DNA. ${ }^{25,29,34}$ However, some effects might be attributed to immune system modulation and changes in tumour blood flow. . $7,35-36^{-3}$

Thus far, electroporation has been used to increase antitumour effectiveness of platinum (II) analogue 3P-SK containing squarato $(\mathrm{skv}=3,4$-dioxocyclobut-1-ene-1,2-diolate) and 3-Hmpy ligands (3P-SK, $\left.\left[\mathrm{Pt}(3-\mathrm{Hmpy})_{2}(\mathrm{skv})\right]\right)$. Indeed, 3P-SK electrochemotherapy treatment had a profound antitumour effectiveness in mouse MCA mammary carcinoma, demonstrating 3P-SK as the first biologically active squarato compound with two 3-Hmpy ligands in vivo. ${ }^{19}$

The promising cytotoxic and antitumour effectiveness of compound 2 from a previous study ${ }^{21}$ encouraged us to assess the electroporation, as drug delivery method, for compound 2 in in vitro and in vivo tumour models with different intrinsic sensitivities to cisplatin. In vitro, clonogenic assays were performed to demonstrate the cytotoxicity of compound $\mathbf{2}$. The cytotoxicity of compound $\mathbf{2}$ alone or in combination with electroporation was investigated in sarcoma cisplatin-sensitive TBLCl2 cells and the cisplatin-resistant subclone TBLCl2Pt, and in sarcoma SA-1 cells with moderate sensitivity to cisplatin, based on in vitro data. ${ }^{25,37}$ Furthermore, the antitumour effectiveness of compound 2 electrochemotherapy using tumour growth delay assay was determined in the same sarcoma tumour models in vivo. In addition, the animal body weight loss was monitored to estimate the influence of the intratumoural administration of compound 2 and local application of electric pulses to the tumour on animal wellbeing. To clarify the underlying mechanism of antitumour effectiveness of compound 2 electrochemotherapy, the amount of platinum in serum and the platinum uptake into tumours, and platinum binding to DNA were measured.

\section{Materials and methods}

\section{Drugs}

Cisplatin (CDDP, Cysplatyl, Aventis Laboratory, Paris, France) was dissolved in sterile water at concentration of $10 \mathrm{mM}$ and frozen in aliquots at $-20^{\circ} \mathrm{C}$ until further use. The compound 2 (trans$\left[\mathrm{PtCl}_{2}(3-\mathrm{Hmpy})_{2}\right]$ was synthesized at the Faculty of Chemistry and Chemical Technology, University of Ljubljana (Ljubljana, Slovenia) ${ }^{21}$ and dissolved in N,N-dimethyl-formamide (DMF, Sigma-Aldrich Co. LCC, St. Louis, MO) at a concentration of 10 $\mathrm{mM}$ and frozen in aliquots at $-20^{\circ} \mathrm{C}$ until further use. 
In the in vitro experiments, concentrations of CDDP and compound 2 ranging from $1.7 \mu \mathrm{M}$ to $1333 \mu \mathrm{M}$ were prepared in a sterile electroporation buffer (EP buffer, $125 \mathrm{mM}$ sucrose, $10 \mathrm{mM} \mathrm{K}_{2} \mathrm{HPO}_{4^{\prime}}$ $2.5 \mathrm{mM} \mathrm{KH}_{2} \mathrm{PO}_{4}$, and $2 \mathrm{mM} \mathrm{MgCl}$ × $6 \mathrm{H}_{2} \mathrm{O}$ ) immediately prior to the experiments.

In the in vivo experiments, fresh solutions of CDDP and compound 2 were prepared in sterile $\mathrm{NaCl}$ solution $(0.9 \%)$ at a final concentration of $13.3 \mathrm{mmol} / \mathrm{kg}$.

\section{Tumour cell lines}

Mouse SA-1 sarcoma cells (Jackson Laboratory, Bar Harbor, ME, USA), mouse TBLCl2 sarcoma cells and the cisplatin-resistant subclone TBLCl2Pt (generously provided by J. Belehradek of the Institute Gustave Roussy, Villejuif, France) $)^{38}$ were used for the in vitro experiments. The SA-1, TBLCl2 and TBLCl2Pt cells were cultured in a humidified incubator at $37^{\circ} \mathrm{C}$ and $5 \% \mathrm{CO}_{2}$ in advanced minimum essential media (AMEM, Gibco, Life Technologies Corporation, Grand Island, NY, USA), supplemented with $5 \%$ of foetal bovine serum (FBS, Gibco). Two days after sub-culturing, the cells in the exponential phase of growth were obtained and used for the cytotoxicity experiments or for the induction of subcutaneous tumours.

\section{Animals and tumour models}

Animal experiments were conducted in accordance with the Veterinary Administration of the Republic of Slovenia (permission No: 3440110/2009/6). Inbreed A/J and C57Bl/6 mice, females 10-12 weeks old, were purchased from the Institute of Pathology, Medical Faculty, University of Ljubljana (Ljubljana, Slovenia) and acclimated to the facility for 10 days. The mice were maintained under specific pathogen-free conditions, at a constant room temperature in a 12-hour day/night light cycle. Food and water were provided ad libitum. Subcutaneous tumours were induced via the injection of $100 \mu \mathrm{L}$ of cell suspension in the right shaved flank of mice; injection of SA-1 sarcoma tumour cell suspension $\left(5 \times 10^{6}\right.$ cells $/ \mathrm{mL}$; obtained from ascites of the donor animal) in A/J mice; injection of TBLCl2 sarcoma $\left(25 \times 10^{6}\right.$ cells/mL; obtained in vitro) and TBLCl2Pt cell suspension $\left(40 \times 10^{6}\right.$ cells/ $\mathrm{mL}$; obtained in vitro) in $\mathrm{C} 57 \mathrm{Bl} / 6$ mice.

\section{Cytotoxicity of electrochemotherapy in vitro}

In the experiments, variable groups were used: control (untreated cells), CDDP or compound
2 (5 minutes incubation of cells with drug), electroporation (EP, cells exposed to application of electric pulses), electrochemotherapy (ECT, cells exposed to combination of EP and drug, CDDP or compound 2). Briefly, cell suspensions of SA-1, TBLC12 and TBLC12Pt sarcoma cells in EP buffer at concentration $2.2 \times 10^{7}$ cells $/ \mathrm{mL}$ were prepared via the trypsinization (Trypsin, Gibco) of two day-old monolayers. The cytotoxicity of treatments was determined using a clonogenic assay. In the EP group, $5 \mu \mathrm{L}$ of EP buffer was added to $45 \mu \mathrm{L}\left(1 \times 10^{6}\right)$ of cell suspension and subsequently placed between two parallel stainless-steel plate electrodes at a 2-mm distance and thereafter exposed to 8 electric pulses (electric field over distance ratio of $1300 \mathrm{~V} / \mathrm{cm}$, duration time of each pulse $100 \mu \mathrm{s}$, at frequency of $1 \mathrm{~Hz}$; generated at Jouan GHT 1287, St. Herblain, France). ${ }^{26,27}$ In CDDP or compound 2 groups, $45 \mu \mathrm{L}$ $\left(1 \times 10^{6}\right)$ of cell suspension was diluted with $5 \mu \mathrm{L}$ of drug solution at a ten times higher final concentration $(1.7 \mu \mathrm{M}-1333 \mu \mathrm{M})$. In the ECT group, $45 \mu \mathrm{L}$ $\left(1 \times 10^{6}\right)$ of cell suspension was diluted with $5 \mu \mathrm{L}$ of drug solution at a ten times higher final concentration $(1.7 \mu \mathrm{M}-1333 \mu \mathrm{M})$ and immediately exposed to electric pulses. Regardless of the treatment, the cells were incubated for 5 minutes at room temperature $\left(22^{\circ} \mathrm{C}\right)$ in an ultra-low attachment 24 -well plate. Subsequently, the cells $(50 \mu \mathrm{L})$ were diluted in $1 \mathrm{~mL}$ of fresh medium and a variable number of cells were seeded onto a Petri dish (diameter $=6$ $\mathrm{cm}$; 200 to 800 cells/Petri dish) for clonogenic assay. In 7-14 days, viable clonogenic cells formed colonies that were stained with crystal violet $(0.005 \%$, Sigma, St. Louis, USA), and only the colonies containing at least 50 cells were counted. The experiment was repeated three times, and each group comprised three replicates.

\section{Electrochemotherapy of tumours in vivo}

The antitumour effectiveness of electrochemotherapy was evaluated using a tumour growth delay assay. When subcutaneous tumours grew to a volume of $40 \mathrm{~mm}^{3}$ in approximately 7-14 days, the animals were divided randomly in experimental groups: control (untreated tumours), CDDP or compound 2 group (intratumoural injection of 50 $\mu \mathrm{L}$ drug solution, CDDP or compound 2), EP (local application of 8 electric pulses in two perpendicular directions (4+4), with electric field over distance ratio of $1300 \mathrm{~V} / \mathrm{cm}, 100 \mu \mathrm{s}$ long, $1 \mathrm{~Hz}$, using two plate parallel stainless-steel electrodes $7 \mathrm{~mm}$ apart placed percutaneously at the opposite margins of tumour; generated by Jouan GHT 1287, 
St. Herblain, France) and combined treatment, electrochemotherapy, i.e., 60 seconds after intratumoural injection of $50 \mu \mathrm{L}$ drug solution electric pulses were applied. Each experimental group comprised 6 to 12 animals. The antitumour effectiveness of therapies was followed by measuring three orthogonal tumour diameters $(a, b, c)$ every other day after therapy using a Vernier calliper and the tumour volume was subsequently calculated using the formula $a \times b \times c \times \pi / 6$. The tumour doubling time for each experimental group based on, tumour growth delay (the tumour doubling time for each experimental group subtracted from the tumour growth delay of control group), was determined as the endpoint of antitumour effectiveness of electrochemotherapy with cisplatin or compound 2. Growth of treated tumours was followed up to a volume of $350 \mathrm{~mm}^{3}$ or in the case of completely regressed tumours, up to 100 days after the treatment (complete response, CR). At these times, the animals were humanely sacrificed via cervical dislocation. Potential side effects of therapies were determined after weighing the animals and assessing behaviour and locomotion.

\section{Platinum determination in tumour and serum}

Measurements of platinum in the serum, tumours and platinum bound to the DNA in the cells were performed using inductively coupled plasma mass spectrometry (ICP-MS, Agilent Technologies, model 7700x, Tokyo, Japan), which monitored the ${ }^{195} \mathrm{Pt}$ isotope. To determine platinum accumulation in the serum at different time points after treatment (3 minutes, 1 hour, 8 and 24 hours), the blood was collected using a glass capillary from intraorbital sinus (8 samples per group) and coagulated at room temperature for two hours. Subsequently, the blood was centrifuged at $3000 \mathrm{rpm}$ for 10 minutes, and the serum was collected and stored at $-20^{\circ} \mathrm{C}$. Furthermore, the total volume of serum samples was digested in $1 \mathrm{~mL}$ of $1: 1$ mixture of $65 \%$ nitric acid (MERCK KgaA, Darmstadt, Germany) and 30\% hydrogen peroxide (MERCK KgaA, Darmstadt, Germany) at $90^{\circ} \mathrm{C}$ for 48 hours. The obtained clear solutions were diluted with Milli-Q water and analysed using ICP-MS.

To obtain tumour samples for platinum determination, the animals were humanely sacrificed immediately after the blood was collected. Tumours were excised (8 samples per group), removed from the overlying skin, weighed and stored at $-20^{\circ} \mathrm{C}$. Subsequently, the tumours were digested in $2 \mathrm{~mL}$ of $1: 1$ mixture of $65 \%$ nitric and $30 \%$ hydrogen peroxide) at $90^{\circ} \mathrm{C}$ for 48 hours, diluted with Milli-Q water and analysed using ICP-MS.

For measurements of platinum binding to the DNA in the tumour cells, the tumours were obtained as described above. However, the tumours were weighed, immediately mechanically degraded and washed with $3 \mathrm{~mL}$ of freshly prepared PBS. Collected samples were filtered through a cell strainer with a pore size of $40 \mu \mathrm{m}$ (Corning Incorporated, Life Sciences, Durham, USA) and centrifuged at $1500 \mathrm{rpm}$ for 10 minutes. The collected cells were used for the fast DNA isolation using a salting-out protocol. First, the cells were lysed with lysis buffer (10 mM Tris- $\mathrm{HCl}, 1 \mathrm{mM}$ EDTA, and 1\% SDS) containing proteinase $\mathrm{K}(0.2 \mu \mathrm{g} / \mathrm{mL})$ at $55^{\circ} \mathrm{C}$ and constant shaking for 30 minutes. The proteins in the cooled samples were precipitated using 120 $\mu \mathrm{L}$ of $4 \mathrm{M} \mathrm{NaCl}$ and shaking for 15 seconds. The precipitated proteins were two times centrifuged at $13000 \mathrm{rpm}$ for 6 minutes. DNA was precipitated from the obtained supernatant using $1 \mathrm{~mL}$ of ethanol $(70 \%)$ for 2 minutes by gentle mixing of tube and centrifugation at $13000 \mathrm{rpm}$ for 2 minutes. The precipitated DNA was washed with an additional $1 \mathrm{~mL}$ of ethanol (70\%) and centrifuged at 13000 rpm for 2 minutes. The pellet of DNA was dried, dissolved in $100 \mu \mathrm{L}$ of distilled water. The concentration of DNA in the samples was spectrophotometrically determined at $260 \mathrm{~nm}$ (Epoch, Take3, Bio Tek Germany). Finally, the samples of DNA were digested using the same procedure described above and the platinum binding to DNA was determined in the diluted samples using ICP-MS.

\section{Statistical analysis}

Statistical analysis was performed using SigmaPlot software (Systat Software, Chicago, IL, USA). The Shapiro-Wilk test was used to determine the normality of data distribution. The differences between mean values of experimental groups were tested using the t-test or one-way ANOVA, followed by the Holm-Sidak test for multiple comparisons. Values of $\mathrm{p}<0.05$ were considered significant.

\section{Results and discussion}

Recently, discovered biological activity of trans platinum complexes with planar aromatic $\mathrm{N}$-donor base $^{21}$, encouraged us to examine compound 2 with electroporation (electrochemotherapy) as a drug delivery approach in tumour cells with different 
TABLE 1. IC 50 values after electrochemotherapy with cisplatin or compound 2 in various mouse tumour cell lines

\begin{tabular}{|c|c|c|c|c|c|c|}
\hline \multirow[b]{2}{*}{ Group } & \multicolumn{2}{|c|}{ SA-1 } & \multicolumn{2}{|c|}{ TBLCl2 } & \multicolumn{2}{|c|}{ TBLCl2Pt } \\
\hline & $\mathrm{IC}_{50}(\mu \mathrm{M})$ & $\mathrm{EF}$ & $I C_{50}(\mu M)$ & EF & $I C_{50}(\mu M)$ & EF \\
\hline Compound 2 & $500.0^{*}$ & & $220.0^{*}$ & & $1066.6^{*}$ & \\
\hline ECT CDDP & $28.3^{\circ}$ & 9.8 & $8.0^{\circ}$ & 22.5 & $12.0^{*}$ & 77.2 \\
\hline
\end{tabular}

$I_{50}=$ dose of drug which reduced cell survival to $50 \%$; $E F=$ enhancement factor for electroporation of cells, calculated on the bases of $I C$ of electroporated and nonelectroporated cells; $\neq \mathrm{p}(<0.05)$ statistically significant difference compared to treatment with cisplatin electrochemotherapy (ECT CDDP); * ${ }_{50}(<0.05)$ significant difference compared to treatment with compound $\mathbf{2}$ electrochemotherapy (ECT Compound 2 ); $p(<0.05)$ statistically difference compared to treatment with compound $\mathbf{2}$ electrochemotherapy.

intrinsic sensitivities to cisplatin in vitro and tumour models in vivo. In vitro, compound 2 electrochemotherapy was effective in cisplatin-sensitive and cisplatin-resistant cells. However, its cytotoxic effect (at the $\mathrm{IC}_{50}$ level) was less evident compared to cisplatin electrochemotherapy, with up to 4-fold in cisplatin-sensitive TBLCl2 cells, up to 1.6-fold in moderately cisplatin-sensitive SA-1 cells and up to 15-fold in cisplatin-resistant TBLC12Pt cells. Similar to in vitro data, growth of tumours with variable intrinsic sensitivity to cisplatin (sensitive, moderately sensitive and resistant) after compound 2 electrochemotherapy was less delayed (up to 9.7 days) compared to cisplatin electrochemotherapy. As expected, the most evident antitumour effectiveness of compound 2 and cisplatin electrochemotherapy was obtained in cisplatin-sensitive TBLCl2 tumours, resulting in $11 \%$ and $67 \%$ of cured tumours, respectively. However, compound 2 electrochemotherapy induced less animal body weight loss compared to cisplatin electrochemotherapy and considering its antitumour effectiveness could be potentially used in further investigation.

\section{Electrochemotherapy with cisplatin or compound 2 efficiently decreased cell survival, in vitro}

Cytotoxicity of platinum compound with 3-hydroxymethilpyridine ligands in cis and trans position has previously been demonstrated in various human and mouse tumour cells. ${ }^{19-21}$ First, in mouse B16 melanoma cells, cis platinum (II) compound with acyclovir (acv, (9-[(2-hydroxyetoxy)methyl] guanine $)$ and 3-Hmpy $\left(\left[\mathrm{Pt}\left(\left[(\mathrm{acv}-\mathrm{N} 7)_{2}(3-\mathrm{Hmpy})_{2}\right]\right.\right.\right.$ $\left.\left(\mathrm{NO}_{3}\right)_{2}\right)$ was 1600 -fold less cytotoxic compared to cisplatin. Furthermore, an increase of cytotoxicity in cisplatin-sensitive and cisplatin-resistant human tumour cell lines was obtained using another platinum (II) compound 3P-SK containing 3-Hmpy and squarato ligands. ${ }^{19}$ However, the enhancement of cytotoxicity was less pronounced compared to cisplatin, particularly in cisplatin-sensitive cells, with a 42-fold enhancement in MCF7 mammary carcinoma, and a 10-fold enhancement in T24 bladder carcinoma and IGROV ovarian carcinoma cells. The compound 3P-SK was least effective in cisplatinresistant IGROV/RDDP ovarian carcinoma cells, showing only a 3-fold increase in $\mathrm{IC}_{50}$ value compared to cisplatin. ${ }^{19}$ Further synthesis of platinum analogues with 3-hmpy ligands led to the discovery of biological active platinum (II) compound with trans geometry possessing two 3-Hmpy ligands, compound 2. Recently, compound $\mathbf{2}$ was demonstrated as equally cytotoxic in various human cisplatin-sensitive T24 bladder carcinoma cells and IGROV 1 ovarian carcinoma cells, and in cisplatinresistant IGROV 1/RDDP ovarian carcinoma cells compared to cisplatin. ${ }^{21}$ In the present study, the cytotoxicity of compound 2 using clonogenic assay was determined in another pair of cisplatinsensitive and cisplatin-resistant cells, TBLCl2 and TBLC12Pt, respectively. Similarly, equal sensitivity of cisplatin-sensitive TBLCl2 and cisplatin-resistant TBLCl2Pt cells was observed, suggesting that compound 2 partially overcomes the mechanisms of resistance to cisplatin (Table 1, Figure 1). Thus, compound 2 could be potentially used in treatment to cisplatin resistant tumours alone or combined with other treatments, such as irradiation, in combination with other chemotherapeutics.

Knowing that cell membrane is the main barrier for drug import in cells, electroporation as the drug delivery method (electrochemotherapy) 

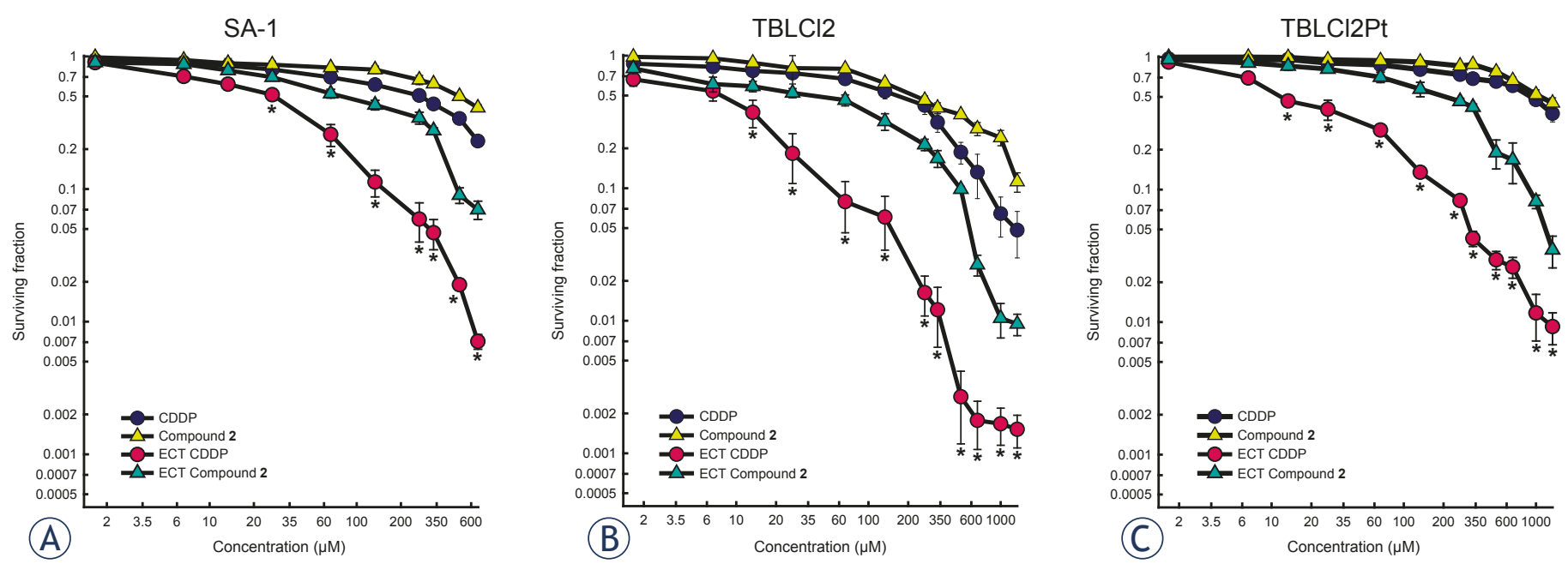

FIGURE 1. Survival of SA-1 sarcoma (A), TBLCl2 sarcoma (B) and TBLCI2Pt sarcoma cells (C) after treatment with cisplatin (CDDP) or compound 2 or cisplatin electrochemotherapy (ECT CDDP) or compound $\mathbf{2}$ electrochemotherapy (ECT Compound 2), determined using a clonogenic assay. Data are presented as the arithmetic mean and standard error of the mean (AM $\pm S E$ ) of triplicates pooled from three independent experiments. The survival of cells treated with electrochemotherapy was normalized to the survival of cells treated with electric pulses alone. The survival of SA-1, TBLCI2 and TBLCI2Pt cells treated with electric pulses alone was $0.96 \pm 0.05,0.93 \pm 0.09$ and $0.92 \pm 0.10$, respectively.

could be used for increasing the amount of compound 2 in the cell, thereby achieving the increased cytotoxic effectiveness of compound 2. The cytotoxicity of cisplatin or compound $\mathbf{2}$ electrochemotherapy was evaluated using a clonogenic assay in cell lines with different intrinsic sensitivities to cisplatin (sensitive TBLCl2, moderate sensitive SA-1 and resistant TBLCl2Pt cells) to obtain reliable results for the cytotoxic effect of compound 2 compared to cisplatin. Electrochemotherapy using either drug significantly decreased $\mathrm{IC}_{50}$ value in all tested cell lines. The most potentiated cytotoxic effect of cisplatin electrochemotherapy was observed in cisplatin-resistant TBLCl2Pt cells, resulting in a 77-fold decrease in $\mathrm{IC}_{50}$ value, as previously demonstrated (79-fold). ${ }^{25}$ However, in moderately cisplatin-sensitive SA-1 cells and cisplatin-sensitive TBLCl2 cells, the reduction in $\mathrm{IC}_{50}$ value was less pronounced in cells exposed to cisplatin electrochemotherapy, and the determined decrease in $\mathrm{IC}_{50}$, up to 23 -fold (Table 1), was in the range of previously published data (10.5-fold in SA-1 and 24-fold in TBLCl2 cells). ${ }^{25,37}$ Using electroporation to increase compound 2 uptake into the cells improved cytotoxicity, resulting in an enhancement factor of 6.3-fold in SA-1 cells, 5.4-fold in TBLCl2 and 5.2-fold in TBLCl2Pt cells. However, the potentiation in the cytotoxic effect of compound 2 electrochemotherapy was significantly lower compared to cisplatin electrochemotherapy, but reflecting a similar enhancement factor in cisplatin-resistant TBLCl2Pt and cisplatin-sensitive TBLCl2 cells
(Table 1, Figure 1), compound 2 still represents a promising drug for further testing.

Since electroporation of cells with cisplatin renders cisplatin-resistant TBLCl2Pt cells to equal level of sensitivity as cisplatin-sensitive cells, and $\mathrm{IC}_{50}$ values were statistically non-significant, consistent with the data obtained in a previous study. ${ }^{25}$ These data indicate increased membrane permeability as the predominant mechanism for the improved cytotoxicity of cisplatin. In fact, the same amount of platinum in both, cisplatin-sensitive $\mathrm{TBLCl} 2$ or cisplatin-resistant TBLCl2Pt cells induced equal cell killing. ${ }^{25}$ Furthermore, the platinum content measured after electrochemotherapy with cisplatin or short 5 minutes incubation with cisplatin alone was comparable in both, cisplatin-sensitive TBLCl2 and cisplatin-resistant TBLCl2Pt cells. ${ }^{25}$ In contrast, significant higher $\mathrm{IC}_{50}$ value determined after compound 2 electrochemotherapy (5-fold higher) in cisplatin-resistant TBLCl2Pt cells compared to cisplatin-sensitive TBLCl2 cells suggests the involvement of other mechanisms besides membrane permeability, such as transport mechanisms (influx or efflux of drug), increased level of thiol molecules in cells, enhanced DNA repair, tolerance to DNA damage and cell death decrease. ${ }^{4-6}$ The function of compound $\mathbf{2}$ differs from that of cisplatin. Compound 2 induces severe conformational changes in plasmid DNA, suggesting that once in the cell, compound 2 likely induces few reparable or irreparable DNA damages, which are reflected in delayed apoptosis. ${ }^{21}$ 
TABLE 2. Antitumour effectiveness of electrochemotherapy with cisplatin or compound 2 in mouse sarcoma tumours; SA-1, TBLCl2 and TBLCl2Pt

\begin{tabular}{|c|c|c|c|c|c|c|c|c|c|c|c|c|}
\hline \multirow[b]{2}{*}{ Group } & \multicolumn{4}{|c|}{ SA-1 } & \multicolumn{4}{|c|}{ TBLCl2 } & \multicolumn{4}{|c|}{ TBLCl2Pt } \\
\hline & $\mathbf{n}$ & $\begin{array}{l}\text { DT (days) } \\
\text { (AM } \pm S E \text { ) }\end{array}$ & $\begin{array}{c}\text { GD } \\
\text { (days) }\end{array}$ & $C R(n, \%)$ & $\mathbf{n}$ & $\begin{array}{l}\text { DT (days) } \\
\text { (AM } \pm S E)\end{array}$ & $\begin{array}{c}\text { GD } \\
\text { (days) }\end{array}$ & CR (n, \%) & $\mathbf{n}$ & $\begin{array}{l}\text { DT (days) } \\
\text { (AM } \pm S E)\end{array}$ & $\begin{array}{c}\text { GD } \\
\text { (days) }\end{array}$ & $C R(n, \%)$ \\
\hline Control & 10 & $1.7 \pm 0.2$ & & 0 & 8 & $2.8 \pm 0.3$ & & 0 & 6 & $3.1 \pm 0.2$ & & 0 \\
\hline EP & 10 & $3.1 \pm 0.3$ & 1.4 & 0 & 8 & $5.1 \pm 1.0$ & 2.3 & 0 & 6 & $4.2 \pm 0.9$ & 1.1 & 0 \\
\hline CDDP & 10 & $5.2 \pm 1.0^{\ddagger}$ & 3.5 & 0 & 8 & $5.3 \pm 0.7^{\ddagger}$ & 2.5 & 0 & 6 & $3.4 \pm 0.5^{\ddagger}$ & 0.3 & 0 \\
\hline Compound 2 & 12 & $2.3 \pm 0.3^{*}$ & 0.6 & 0 & 9 & $3.5 \pm 0.5^{*}$ & 0.7 & 0 & 6 & $3.2 \pm 0.3^{*}$ & 0.1 & 0 \\
\hline ECT CDDP & 12 & $15.8 \pm 2.2^{*}$ & 14.1 & 0 & 9 & $11.6 \pm 1.0$ & 8.8 & $6,66.6$ & 6 & $7.6 \pm 0.9^{*}$ & 4.5 & 0 \\
\hline ECT Compound 2 & 12 & $5.1 \pm 0.4$ & 3.4 & 0 & 9 & $9.4 \pm 1.8$ & 6.6 & $1,11.1$ & 6 & $5.1 \pm 0.6$ & 2.0 & 0 \\
\hline
\end{tabular}

$\mathrm{EP}=$ application of electric pulses; CDDP = cisplatin, intratumoural injection at a dose of $13.3 \mathrm{mmol} / \mathrm{kg}$; Compound 2- intratumoural injection at a dose $13.3 \mathrm{mmol} / \mathrm{kg}$; ECT = electrochemotherapy, application of EP at 1 minute after intratumoural injection of CDDP or compound 2 ; DT = tumour doubling time; GD = tumour growth delay; CR = complete response; $\mathrm{AM}=$ mean; $\mathrm{SE}=$ standard error of the mean; ${ }^{\ddagger} \mathrm{p}(<0.05)$ significant difference compared to treatment with $\mathrm{CDDP}$ electrochemotherapy; ${ }^{\mathrm{p}} \mathrm{P}(<0.05)$ significant difference compared to treatment with compound 2 electrochemotherapy.

\section{Electrochemotherapy with cisplatin or compound 2 efficiently delayed tumour growth in vivo}

Until recently, few trans-Pt(II) complexes were evaluated in vivo. The analogues of transplatin (trans- $\left[\mathrm{PtCl}_{2}\left(\mathrm{NH}_{3}\right)_{2}\right]$ ) with one $\mathrm{NH}_{3}$ exchanged with pyridine or 4-methylpyridine had no effect in the S180 sarcoma tumour model..$^{39}$ In P388 leukaemia an evident antitumour effectiveness was obtained with compound having one iminoether instead of an amine ligand. ${ }^{40}$ However, the replacement of two amine ligands with dimethylamine and isopropylamine failed to improve the antitumour activity in human $\mathrm{CH}$ ovarian carcinoma, reflecting its extracellular inactivation after binding to plasma proteins. ${ }^{41}$ These results indicate the nature of the ligand as an important player in the biological activation of trans-Pt(II) compounds. Recently, we showed the promising biological activity of transPt compound 2 with both amino groups replaced with pyridine derivate containing hydroxyl group, 3-Hmpy. Interestingly, a comparable antitumour effectiveness of treatment with triple intravenously administered compound 2 in sarcoma SA- 1 tumours to cisplatin was obtained. ${ }^{21}$

To evaluate compound 2 as a potential chemotherapeutic drug in combination with electroporation, for the induction of subcutaneous tumours, the same mouse sarcoma tumour cell lines as used in vitro (moderately cisplatin-sensitive SA1 cells, cisplatin-sensitive TBLCl2 and -resistant TBLCl2Pt cells) were selected. Since in vitro data have shown sarcoma cells as resistant to compound 2 (high $\mathrm{IC}_{50}$ ), the purpose of the research was to evaluate whether the intrinsic resistance of these cells is reflected in tumours in vivo. The route (intratumoural or intravenous) of drug administration plays an important role in drug distribution and toxicity to normal tissues. In some cases, electrochemotherapy with intratumoural injection of cisplatin showed evident antitumour potential over systemic administration, reflecting a higher concentration of the drug obtained in tumours and lower concentration in normal tissues during electroporation. ${ }^{34,42}$ Furthermore, compound 2 pharmacologically behaves differently compared to cisplatin, and more platinum in serum and tumour was observed. ${ }^{21}$ According to these observations, in the present study, the single intratumoural administration of drug was used to potentially induce less side effects of the treatment and achieve the highest drug concentration in the tumours at the time of electroporation. In general, the antitumour effectiveness of treatment with compound 2 alone was less pronounced compared to cisplatin in all tumour models with intrinsically different sensitivity to cisplatin (Table 2, Figure 2). Treatment of moderately cisplatin-sensitive SA-1 tumours with cisplatin alone resulted in a significant delay of tumour growth compared to compound 2. Regarding the route of drug administration in SA-1 tumours, triple intravenous ${ }^{21}$ or single intratumoural injection resulted in comparable antitumour effectiveness of cisplatin alone. However, the delay of tumour growth after triple intravenous administration of compound $\mathbf{2}^{21}$ was more pronounced (up to 2.2 days) compared to single intratumoural administration of compound 2 in the present study. Furthermore, antitumour 

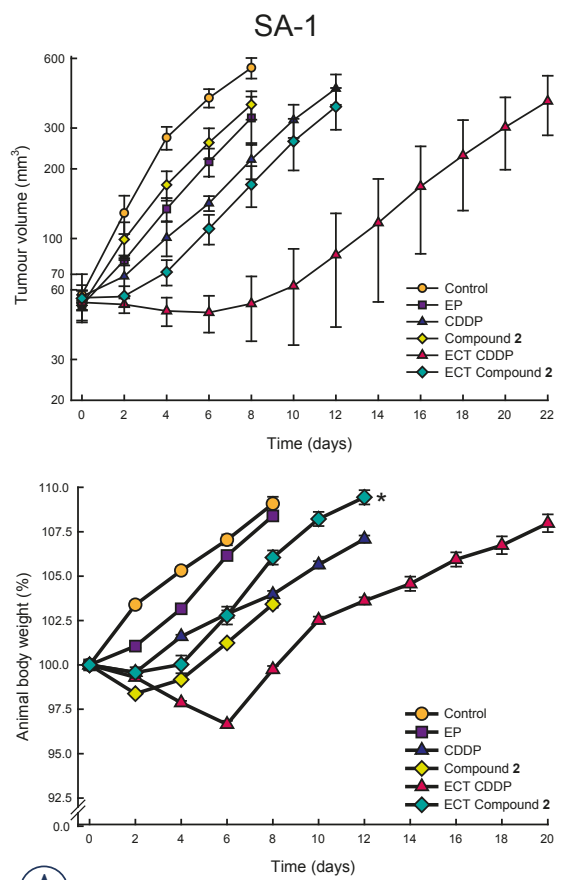

(A)
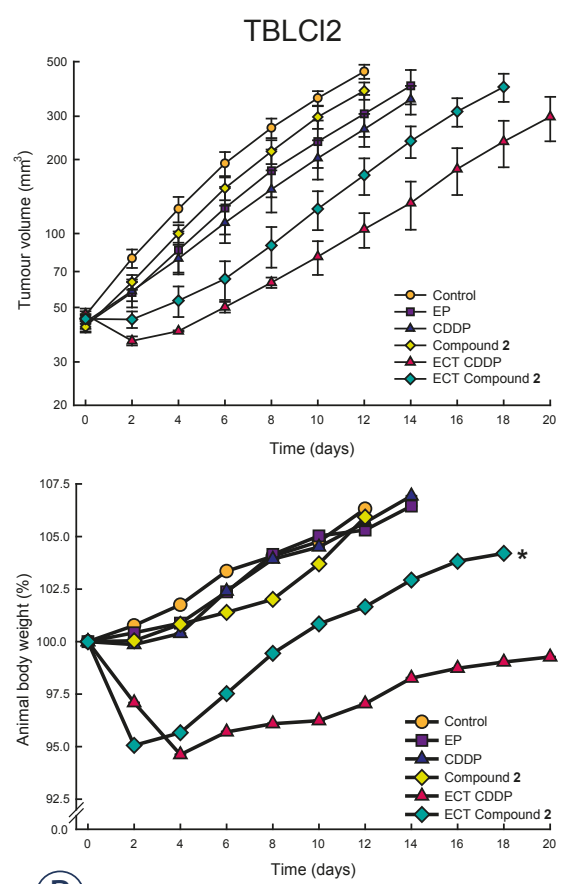

(B)
TBLCl2Pt
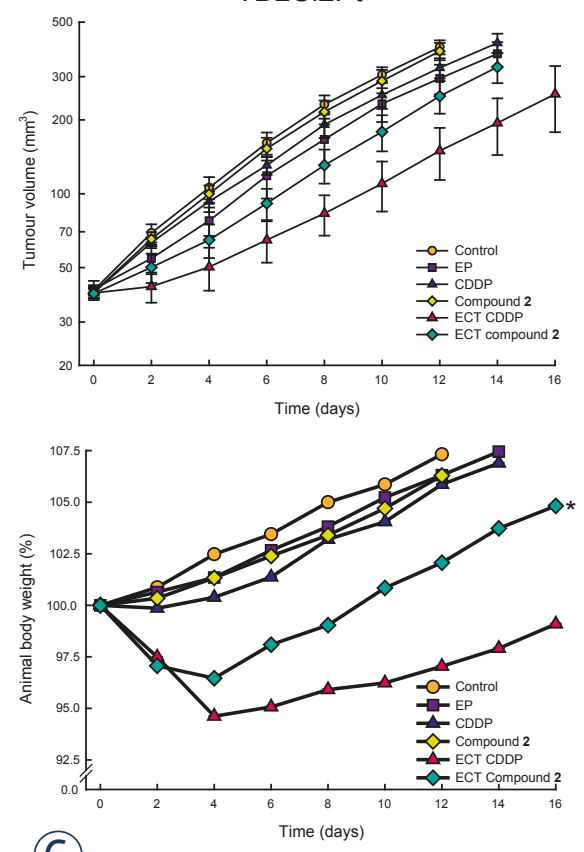

(C)

FIGURE 2. Tumour growth and animal body weight change after cisplatin or compound 2 electrochemotherapy in mouse sarcoma tumours; SA-1, TBLCl2 and TBLCl2Pt. Mice (6-12 per group) were treated with intratumoural injection of cisplatin (CDDP, $13.3 \mathrm{mmol} / \mathrm{kg}$ ) or compound 2 (13.3 mmol/ $/ \mathrm{kg}$ ) or with local application of electric pulses at 1 minute after drug injection (ECT CDDP; ECT Compound 2; 8 pulses, $1300 \mathrm{~V} / \mathrm{cm}, 100 \mu \mathrm{s}, 1 \mathrm{~Hz}$ ). Data are presented as the arithmetic mean and standard error of the mean ( $A M \pm S E$ ) of tumour volumes." $\mathrm{p}(<0.05)$ significant difference compared to treatment with cisplatin electrochemotherapy

effectiveness was less evident in cisplatin-sensitive TBLCl2 and cisplatin-resistant TBLCl2Pt as in moderately cisplatin-sensitive SA-1 tumours, indicating different intrinsic features of the tumour models examined, particularly the mechanisms of resistance to cisplatin. Several efforts to overcome the mechanisms of cisplatin resistance, i.e., using approaches to decrease glutathione level, affect DNA repair, activate signal transduction pathways leading to cell death and increase cisplatin accumulation, have been used.5,17,23,25,43-46 The predominant mechanism of cisplatin resistance in TBLC12Pt cells was suggested as the membrane restriction of cisplatin uptake. ${ }^{25}$ Thus, electroporation could be used to overcome the resistance to cisplatin and potentiate antitumour effectiveness of cisplatin, as previously demonstrated in variable tumour models. ${ }^{19,25,28,34,37,42}$ Application of electric pulses to the tumours alone insignificantly delayed tumour growth in all tested tumour models and the data are consistent with previous studies. ${ }^{25,28,38}$ However, combined treatment of intratumoural injection either of cisplatin or compound 2 shortly before application of electric pulses, significantly potentiated (up to 3-fold) antitumour effectiveness in all tumour models, but less prominent effects were observed with compound 2. As expected, most pronounced antitumour effectiveness after single electrochemotherapy treatment was obtained in cisplatin-sensitive TBLCl2 tumours, resulting in $67 \%$ tumour cures after cisplatin electrochemotherapy and $11 \%$ tumour cures after compound 2 electrochemotherapy. Notably, cisplatin or compound 2 alone did not delay growth of cisplatin-resistant TBLCl2Pt tumours, but electroporation potentiated antitumour effectiveness of either of the drugs used, resulting in prolonged tumour 2.2-fold and 1.6-fold growth delay, respectively (Table 2, Figure 2). These data suggest that compound 2 electrochemotherapy as an appropriate alternative therapy in cisplatin-resistant tumours or tumours with acquired cisplatin-resistance. Studies using electroporation to improve the antitumour effectiveness of newly synthesized chemotherapeutic are scarce. Among the reported studies, two ruthenium (III) compounds, KP418 ((imH)[trans- $\left.\mathrm{RuCl}_{4}(\mathrm{im})_{2}\right], \quad \mathrm{im}=$ imidazole $)$ and KP1339 (Na[trans-RuCl $\left.{ }_{4}(\mathrm{in})_{2}\right]$, in=indazole), administered intravenously, were used in combination with electroporation. ${ }^{47-48}$ However, significant antitumour effectiveness was only obtained with KP1339 electrochemotherapy in SA-1 tumours, 
while electrochemotherapy with KP418 has no significant effect on tumour growth delay in SA-1 and in B16F1 melanoma tumours. Although, the type of administration of KP1339 was different (intravenously), at equimolar doses, its antitumour effectiveness was comparable to the effect of compound 2. To our knowledge, only one synthesized platinum (II) compound with 3-Hmpy ligands, 3P-SK, was used in combination with electroporation in recent study of our research group. ${ }^{19}$ In that study, similar to the results of the present study, the antitumour effectiveness of local application of electric pulses after intratumoural administration of 3P-SK was less pronounced compared to cisplatin. Still, 3P-SK electrochemotherapy significantly reduced growth of mouse MCA mammary carcinoma tumours and cured $14 \%$ of tumours. ${ }^{19}$ Overall, considering the 3-fold potentiation in antitumour effectiveness of compound 2 electrochemotherapy compared to the 2-fold potentiation obtained after 3P-SK electrochemotherapy ${ }^{19}$, compound 2 may potentially be more biologically active. Moreover, the potentiation level in antitumour effectiveness of compound 2 electrochemotherapy compared to cisplatin electrochemotherapy, varied between 1.6- and 3-fold, suggesting that different transport mechanisms and intracellular mechanisms of action between compound 2 and cisplatin could be involved in tumour responses (Table 2, Figure 2).

In addition, animal body weight, behaviour and locomotion as indicators of animal wellbeing were monitored after cisplatin or compound 2 electrochemotherapy. Single treatment with intratumoural administration of compound $\mathbf{2}$ and application of electric pulses induced significantly less body weight loss compared to cisplatin. The animals showed a loss of body weight up to 6 days after treatment (approximately 6\%) and recovered thereafter (Figure 2). These results are consistent with those of a previous study, showing that animals bearing SA-1 sarcoma lost significantly less body weight after triple intravenous administration of compound 2 compared to cisplatin. ${ }^{21}$ However, the body weight loss obtained after single intratumoural administration of compound 2 compared to triple intravenous administration of compound 2 was less pronounced, indicating that the intratumoural administration of compound 2 was less toxic. Since electrochemotherapy with compound 2 demonstrates fewer side effects on animal wellbeing and despite less pronounced antitumour effectiveness compared to cisplatin, further studies are warranted, particularly for testing the repetitive treatment of tumours with electrochemotherapy.

\section{Electrochemotherapy with cisplatin or compound 2 increases platinum uptake in tumour and amount of platinum bound to DNA}

To clarify whether the antitumour effectiveness of electrochemotherapy either with compound 2 or cisplatin was consistent with increased uptake of the drug in the cells and partially clarify the pharmacology of the drugs used, the amount of platinum was measured in tumours, serum and bound to the DNA. Previous studies have determined an increase in platinum accumulation after application of electric pulses in various mouse tumours (sarcoma (SA-1, LPB) and carcinoma (EAT) tumours), which consequently improve the antitumour effectiveness of cisplatin..$^{27,29,34}$ To prevent the washout of drug from the tumour and ensure the highest concentration in SA-1 tumours, electric pulses were delivered at 1 minute after intratumoural injection, as previously optimized in an EAT tumour model. ${ }^{34}$ Measurement of platinum amount using ICPMS in the present study demonstrated electroporation as an effective delivery method for compound 2 , transplatin analogue, and cisplatin. An approximately significant 2 -fold increase of platinum in tumours was achieved, resulting in pronounced antitumour effectiveness (Figure 3). Initial level of platinum amount in tumours treated with compound 2 alone or compound 2 electrochemotherapy was 1.3-fold higher compared to cisplatin. In addition, the wash out of platinum from the tumours treated with cisplatin or compound 2 alone was up to 1.5-fold quicker compared to tumours treated with electrochemotherapy using either of the drugs. The initial platinum amount in the tumours at one hour after the treatment with drug alone or with electrochemotherapy was reduced up to $48 \%$ and up to $18 \%$, respectively, indicating the binding of tested drug to the proteins in serum, in extracellular tumour matrix and transport into the cell. In fact, the aromatic ring of pyridine and hydroxyl groups participates in non-covalent interactions between platinum compound and DNA.9, 11, 14, 18-20 Furthermore, triple intravenous administration of compound 2 compared to cisplatin resulted in significant higher platinum amount in tumours and serum, 4-fold and 40-fold, respectively. ${ }^{21}$ Similarly, significantly higher concentration (up to 39-fold) of platinum concentration in serum of animals treated either with compound 2 alone or compound 2 electrochemotherapy compared to cisplatin correlated with more platinum in tumours (Figure 3 ). Altogether, compound 2 exhibits a different phar- 
a.)

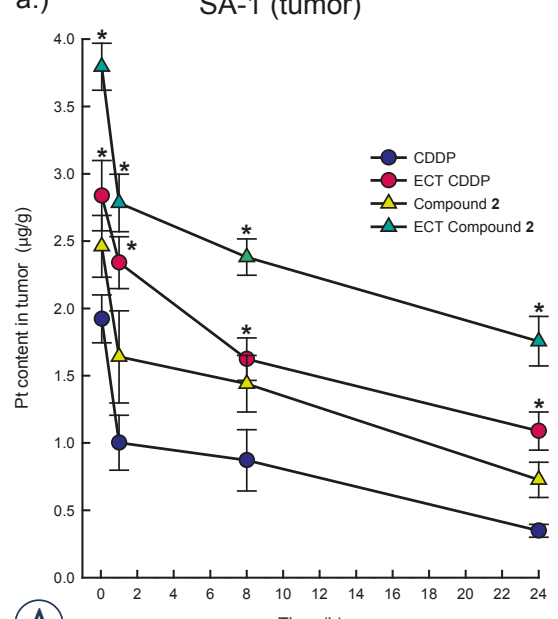

(A) b.)

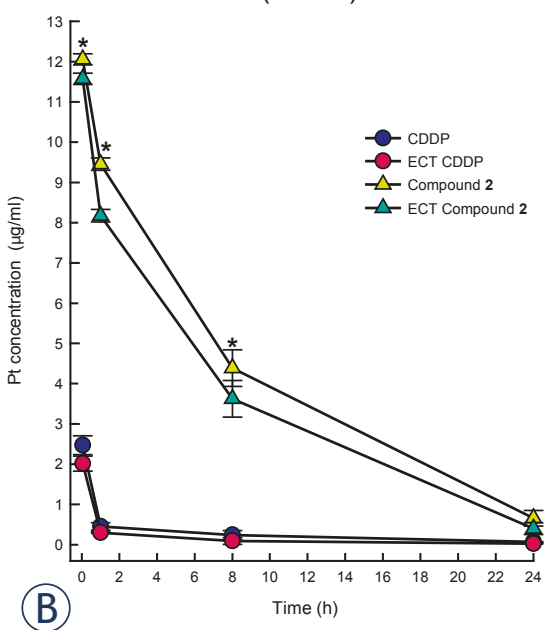

c.)

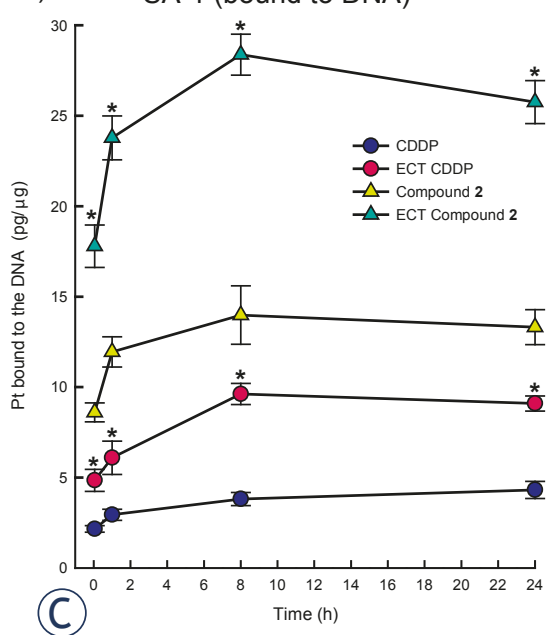

FIGURE 3. Platinum amount in sarcoma SA-1 tumours (A), platinum amount in the serum (B) and platinum amount bound to DNA in the cells isolated from SA-1 tumours (C). Animals (8 per group) were treated with intratumoural injection of cisplatin (CDDP, $13.3 \mathrm{mM}$ ) alone or compound $\mathbf{2}$ (13.3 mM) alone or with local application of electric pulses 1 minute after intratumoural drug injection (ECT CDDP; ECT Compound 2; 8 pulses, $1300 \mathrm{~V} / \mathrm{cm}, 100 \mu \mathrm{s}$, $1 \mathrm{~Hz}$ ). The data are presented as the arithmetic mean and standard error of the mean (AM $\pm S E$ ) obtained from 8 samples. " $p(<0.05)$ under (A) and (C) statistically significant difference compared to corresponding drug treatment only; " $p(<0.05)$ under (B) statistically significant difference compared to cisplatin or cisplatin electrochemotherapy.

macology compared to cisplatin, which could affect tumour responses. Compared to the pharmacology of cisplatin, other mechanisms could be involved in tumour responses to electrochemotherapy. In particular, the drug distribution in tumours depends on tumour vascularization and the content of tumour extracellular matrix, which plays an important role before application of electric pulses. Vascular disruption, reduced oxygenation and induced immune responses greatly contribute to tumour responses. ${ }^{35-36,49-50}$

DNA is considered the main intracellular target of cisplatin and its analogues. Hence, to clarify that antitumour effectiveness of electrochemotherapy primarily depends on increased drug uptake, the amount of platinum bound to DNA was evaluated as an indicator that compound 2 escaped binding to intracellular thiol proteins and reached DNA (Figure 3). Indeed, in tumours treated with electrochemotherapy compared to the drug administration only, approximately 2-fold higher platinum binding to DNA was achieved, correlated with pronounced antitumour effectiveness (Figure 3). Similarly, the higher level of platinum in tumours and serum in animals treated with compound 2 electrochemotherapy compared to cisplatin was reflected in a significantly (approximately 3 -fold) higher amount of platinum bound to DNA. However, the antitumour effectiveness was less evident. Overall, a 4-fold increase of platinum bound to DNA was obtained in tumours treated with compound 2 compared to cisplatin. In vitro, despite the enhanced accumulation of a trans-platinum (II) compounds, either with amine, oxine or piperidine ligand, in tumour cells, the cytotoxicity remained comparable or lower than that of cisplatin. ${ }^{51-53}$ Thus, the increased accumulation and binding capacity to DNA obviously are not crucial for cytotoxic potential of compounds, suggesting the importance of the mode DNA interaction and DNA repair. Thus, depending on the ligand in trans platinum compound a monofunctional and bifunctional adducts can be formed with DNA. ${ }^{9-10,54-56}$ Recently, we demonstrated the formation of severe conformational changes in plasmid DNA after treatment with compound 2, and consistent with the findings using other transplatinum analogues $9,54,56$, the formation of bifunctional DNA crosslinks was suggested. Additionally, the effectiveness of DNA repair mechanisms may also render compound 2 less cytotoxic compared to cisplatin. Moreover, the route of drug administration to some extent affected the level of platinum bound to the DNA. Approximately $14 \%$ more platinum bound to DNA was obtained after intratumoural compared to the intravenous administration of either compound 2 or cisplatin..$^{21}$ Thus, as $80 \%$ of cisplatin in blood circulation is bound to proteins, primarily albumin, this difference could be expected, suggesting less free cisplatin molecules reached tumour nodule. ${ }^{57-58}$ Taken altogether, the pharmacology of compound 2 seems to differ from cisplatin, 
involving binding to the proteins in blood circulation, in tumour extracellular matrix and accumulation in cells. The accumulation of cisplatin in the cell is not fully understood. Cisplatin could enter in cells through passive diffusion and facilitated or active transport mediated through membrane transporters (the Copper transporter 1 (CTR1) and 2 (CTR2); the P-type copper-transporting ATPases ATP7A and ATP7B, multidrug and toxin extrusion transporters). ${ }^{7}$ Currently, little is known about the accumulation of transplatin or its analogues. Active transport was demonstrated to play an important role in accumulation, independent of transporter CTR1 and ATP, depending on protein ATP7B and CTR2. ${ }^{6,8}$ The details of compound 2 transport mechanisms and its interaction with biological molecules and antitumour effectiveness need further investigation.

\section{Conclusions}

In summary, compound 2, a trans-Pt(II) analogue with two 3-Hmpy ligands, shows potential for electrochemotherapy treatment in vitro and in vivo. Electroporation increased compound 2 cytotoxicity up to 6-fold in vitro and antitumour effectiveness up to 3-fold in vivo, but it had less evident effects compared to cisplatin. The underlying mechanism of antitumour effectiveness of electrochemotherapy could be increased drug uptake, and an approximately 2 times higher amount of platinum in the tumours was reflected to the same extent in higher amount of $\mathrm{Pt}$ bound on its target of action to the DNA. Based on the compound 2 electrochemotherapy cytotoxicity and antitumour effectiveness in cisplatin-resistant tumour model TBLCl2Pt, this molecule could potentially be used in the treatment of tumours with intrinsic or acquired resistance to cisplatin. To improve the antitumour effectiveness of compound 2 electrochemotherapy, multiple treatments should be tested in the future. Furthermore, compound 2 could be used in combination with other chemotherapeutics, affecting different targets, immunotherapy, vascular-targeted therapy or irradiation; however, further studies are warranted.

\section{Acknowledgements}

The authors gratefully acknowledge the research grants from the Slovenian Research Agency (P30003 and P1-0134).

\section{References}

1. Emens LA, Middleton $G$. The interplay of immunotherapy and chemotherapy: harnessing potential synergies. Cancer Immunol Res 2015; 3: 436-443. doi:10.1158/2326-6066.CIR-15-0064

2. Riaz N, Sherman E, Koutcher L, Shapiro L, Katabi N, Zhang ZG, et al. Concurrent chemoradiotherapy with cisplatin versus cetuximab for squamous cell carcinoma of the head and neck. Am J Clin Oncol-Canc 2016; 39: 27-31. doi:10.1097/COC.0000000000000006

3. Izgi K, Iskender B, Sakalar C, Arslanhan A, Yuksek EH, Hizar E, et al. Effects of epirubicin and cisplatin against 4T1 breast cancer cells are enhanced by myrtucommulone-A. Anticancer Agents Med Chem 2016. [Epub ahead of print]. doi:10.2174/1871520616666160404110543

4. Amable L. Cisplatin resistance and opportunities for precision medicine. Pharmacol Res 2016; 106: 27-36. doi:10.1016/j.phrs.2016.01.001

5. Galluzzi L, Vitale I, Michels J, Brenner C, Szabadkai G, Harel-Bellan A, et al. Systems biology of cisplatin resistance: past, present and future. Cell Death Dis 2014; 5: e1257. doi:10.1038/cddis.2013.428

6. Burger $\mathrm{H}$, Zoumaro-Djayoon A, Boersma AWM, Helleman J, Berns EMJJ, Mathijssen RHJ, et al. Differential transport of platinum compounds by the human organic cation transporter hOCT2 (hSLC22A2). Brit J Pharmacol 2010; 159: 898-908. doi:10.1111/j.1476-5381.2009.00569.

7. Ciarimboli G. Membrane transporters as mediators of cisplatin side-effects. Anticancer Res 2014; 34: 547-50.

8. Larson CA, Blair BG, Safaei R, Howell SB. The role of the mammalian copper transporter 1 in the cellular accumulation of platinum-based drugs. $\mathrm{Mol}$ Pharmacol 2009; 75: 324-30. doi:10.1124/mol.108.052381

9. Icsel C, Yilmaz VT, Ari F, Ulukaya E, Harrison WTA. trans-Dichloridopalladium (II) and platinum (II) complexes with 2-(hydroxymethyl)pyridine and 2-(2-hydroxyethyl)pyridine: synthesis, structural characterization, DNA binding and in vitro cytotoxicity studies. Eur J Med Chem 2013; 60: 386-94. doi:10.1016/j.ejmech.2012.12.002

10. Kasparkova J, Marini V, Najajreh Y, Gibson D, Brabec V. DNA binding mode of the cis and trans geometries of new antitumor nonclassical platinum complexes containing piperidine, piperazine, or 4-picoline ligand in cell-free media. Relations to their activity in cancer cell lines. Biochemistry 2003; 42: 6321-32. doi:10.1021/bi0342315

11. Martinez A, Lorenzo J, Prieto MJ, Font-Bardia M, Solans X, Aviles FX, et al. Influence of the position of substituents in the cytotoxic activity of trans platinum complexes with hydroxymethyl pyridines. Bioorg Med Chem 2007; 15: 969-79. doi:10.1016/j.bmc.2006.10.031

12. Najajreh Y, Perez JM, Navarro-Ranninger C, Gibson D. Novel soluble cationic trans-diaminedichloroplatinum (II) complexes that are active against cisplatin resistant ovarian cancer cell lines. J Med Chem 2002; 45: 5189-95.

13. Quiroga AG. Understanding trans platinum complexes as potential antitumor drugs beyond targeting DNA. J Inorg Biochem 2012; 114: 106-12. doi:10.1016/j.jinorgbio.2012.06.002

14. Ramos-Lima FJ, Moneo V, Quiroga AG, Carnero A, Navarro-Ranninger C. The role of p53 in the cellular toxicity by active trans-platinum complexes containing isopropylamine and hydroxymethylpyridine. Eur J Med Chem 2010; 45: 134-41. doi:10.1016/j.ejmech.2009.09.035

15. Coluccia M, Natile G. Trans-platinum complexes in cancer therapy. Anticancer Agents Med Chem 2007; 7: 111-23.

16. Johnstone TC, Suntharalingam K, Lippard SJ. The next generation of platinum drugs: targeted Pt (II) agents, nanoparticle delivery, and Pt (IV) prodrugs. Chem Rev 2016; 116: 3436-86. doi:10.1021/acs.chemrev.5b0059

17. Shahsavar F, Bozorgmehr M, Mirzadegan E, Abedi A, Lighvan ZM, Mohammadi $F$, et al. A novel platinum-based compound with preferential cytotoxic activity against a panel of cancer cell lines. Anticancer Agents Med Chem 2016; 16: 393-403.

18. Ari F, Aztopal N, Icsel C, Yilmaz VT, Guney E, Buyukgungor O, et al. Synthesis, structural characterization and cell death-inducing effect of novel palladium (II) and platinum (II) saccharinate complexes with 2-(hydroxymethyl) pyridine and 2-(2-hydroxyethyl) pyridine on cancer cells in vitro. Bioorg Med Chem 2013; 21: 6427-34. doi:10.1016/j.bmc.2013.08.050

19. Cemazar M, Pipan Z, Grabner S, Bukovec N, Sersa G. Cytotoxicity of different platinum (II) analogues to human tumour cell lines in vitro and murine tumour in vivo alone or combined with electroporation. Anticancer Res 2006; 26: $1997-2002$. 
20. Grabner S, Cemazar M, Bukovec N, Sersa G. Syntheses and cytotoxicity of Pt (II) complexes with acyclovir. Acta Chim Slov 2006; 53: 153-8.

21. Grabner S, Modec B, Bukovec N, Bukovec P, Cemazar M, Kranjc S, et al. Cytotoxic trans-platinum (II) complex with 3-hydroxymethylpyridine: synthesis, X-ray structure and biological activity evaluation. J Inorg Biochem 2016; 161: 40-51. doi:10.1016/j.jinorgbio.2016.04.031

22. Tiwari G, Tiwari R, Sriwastawa B, Bhati L, Pandey S, Pandey $P$, et al. Drug delivery systems: an updated review. Int J Pharm Investig 2012; 2: 2-11. doi:10.4103/2230-973X.96920

23. Kaur H, Desai SD, Kumar V, Rathi P, Singh J. Heterocyclic drug-polymer conjugates for cancer targeted drug delivery. Anticancer Agents Med Chem 2016; 16: 1355-77.

24. Neumann E, Schaeferridder M, Wang Y, Hofschneider PH. Gene-transfe into mouse lyoma cells by electroporation in high electric-fields. EMBO J 1982; 1: 841-5.

25. Cemazar M, Miklavcic D, Mir LM, Belchradek J, Bonnay M, Fourcault D, et al. Electrochemotherapy of tumours resistant to cisplatin: a study in a murine tumour model. Eur J Cancer 2001; 37: 1166-72. 10.1016/S09598049(01)00091-0

26. Kranjc S, Cemazar M, Grosel A, Pipan Z, Sersa G. Effect of electroporation on radiosensitization with cisplatin in two cell lines with different chemo- and radiosensitivity. Radiol Oncol 2003; 37: 101-7.

27. Kranjc S; Cemazar M, Grosel A, Scancar J, Sersa G. Electroporation of LPB sarcoma cells in vitro and tumors in vivo increases the radiosensitizing effect of cisplatin. Anticancer Res 2003; 23: 275-81.

28. Sersa G, Cemazar M, Miklavcic D. Antitumor effectiveness of electrochemotherapy with cis-diamminedichloroplatinum (II) in mice. Cancer Res 1995; 55: 3450-5.

29. Cemazar M, Miklavcic D, Scancar J, Dolzan V, Golouh R, Sersa G. Increased platinum accumulation in SA-1 tumour cells after in vivo electrochemotherapy with cisplatin. Brit J Cancer 1999; 79: 1386-91.

30. Cemazar M, Tamzali Y, Sersa G, Tozon N, Mir L.M, Miklavcic D, et al. Electrochemotherapy in veterinary oncology. J Vet Intern Med 2008; 22 826-31. doi:10.1111/j.1939-1676.2008.0117.x

31. Maglietti F, Tellado M, Olaiz N, Michinski S, Marshal, G. Combined local and systemic bleomycin administration in electrochemotherapy to reduce the number of treatment sessions. Radiol Oncol 2016; 50: 58-63. doi:10.1515/ raon-2016-0015

32. Mali B, Jarm T, Snoj M, Sersa G, Miklavcic D. Antitumor effectiveness of electrochemotherapy: a systematic review and meta-analysis. Eur J Surg Oncol 2013; 39: 4-16. doi:10.1016/j.ejso.2012.08.016

33. Yarmush ML, Golberg A, Sersa G, Kotnik T, Miklavcic D. Electroporationbased technologies for medicine: principles, applications, and challeng es. Annu Rev Biomed Eng 2014; 16: 295-320. doi:10.1146/annurev-bioeng-071813-104622

34. Cemazar M, Milacic R, Miklavcic D, Dolzan V, Sersa G. Intratumoral cisplatin administration in electrochemotherapy: antitumor effectiveness, sequence dependence and platinum content. Anticancer Drugs 1998; 9: 525-30.

35. Sersa G, Teissie J, Cemazar M, Signori E, Kamensek U, Marshall Get al. Electrochemotherapy of tumors as in situ vaccination boosted by immunogene electrotransfer. Cancer Immunol Immunother 2015; 64: 1315-27. doi:10.1007/s00262-015-1724-2

36. Markelc B, Sersa G, Cemazar M. Differential mechanisms associated with vascular disrupting action of electrochemotherapy: intravital microscopy on the level of single normal and tumor blood vessels. PloS One, 2013; 8: e59557. doi:10.1371/journal.pone.0059557

37. Belehradek JJ, Barski G, Thonier M. Evolution of cell-mediated antitumor immunity in mice bearing a syngeneic chemically induced tumor. Influence of tumor growth, surgical removal and treatment with irradiated tumor cells. Int J Cancer 1972: 9: 461-9.

38. Sedlar A, Dolinsek T, Markelc B, Prosen L, Kranjc S, Bosnjak M, et al. Potentiation of electrochemotherapy by intramuscular $I L-12$ gene electrotransfer in murine sarcoma and carcinoma with different immunogenicity. Radiol Oncol 2012; 46: 302-11. doi:10.2478/v10019-012-0044-9

39. Hollis L.S, Amundsen AR, Stern EW. Chemical and biological properties of new series of cis-diammineplatinum (II) antitumor agents containing three nitrogen donors: cis-[Pt( $\left.\mathrm{NH}_{3}\right)_{2}(\mathrm{~N}-$ donor $\left.) \mathrm{Cl}\right]^{+}$. J Med Chem 1989; 32: 128-36.

40. Leng $M$, Locker D, Giraud-Panis MJ, Schwartz A, Intini FP, Natile G, et al. Replacement of an $\mathrm{NH}_{3}$ by an iminoether in transplatin makes an antitumor drug from an inactive compound. Mol Pharmacol 2000; 58: 1525-35.
41. Perez JM, Kelland LR, Montero El, Boxall FE, Fuertes MA, Alonso C, et al. Antitumor and cellular pharmacological properties of a novel platinum (IV) complex: trans-[ $\mathrm{PtCl}_{2}(\mathrm{OH})_{2}$ (dimethylamine) (isopropylamine)]. $\mathrm{Mol}$ Pharmacol 2003; 63: 933-44.

42. Cemazar M, Miklavcic D, Vodovnik L, Jarm T, Rudolf Z, Stabuc B, et al Improved therapeutic effect of electrochemotherapy with cisplatin by intratumoral drug administration and changing of electrode orientation for electropermeabilization on EAT tumor model in mice. Radiol Oncol 1995; 29: 121-7.

43. Baharuddin P, Satar, N, Fakiruddin KS, Zakaria N, Lim MN, Yusoff NM, et al. Curcumin improves the efficacy of cisplatin by targeting cancer stem-like cells through p21 and cyclin D1-mediated tumour cell inhibition in nonsmall cell lung cancer cell lines. Oncol Rep 2016; 35: 13-25. doi:10.3892/ or.2015.4371

44. Lee JH, Chae JW, Kim JK, Kim HJ, Chung JY, Kim YH. Inhibition of cisplatinresistance by RNA interference targeting metallothionein using reducible oligo-peptoplex. J Control Release 2015; 215: 82-90. doi:10.1016/j.jconrel.2015.07.015

45. Chong SX, Au-Yeung SC, To KK. Monofunctional platinum (PtII) compounds - shifting the paradigm in designing new pt-based anticancer agents. Curr Med Chem 2016; 23: 1268-85. doi:10.2174/0929867323666160311114509

46. Igarashi K, Yamamoto N, Hayashi K, Takeuchi A, Miwa S, Odani A, et al. Effectiveness of two novel anionic and cationic platinum complexes in the treatment of osteosarcoma. Anticancer Agents Med Chem 2015; 15: 390-9.

47. Hudej R, Miklavcic D, Cemazar M, Todorovic V, Sersa G, Bergamo A, et al. Modulation of activity of known cytotoxic ruthenium (III) compound (KP418) with hampered transmembrane transport in electrochemotherapy in vitro and in vivo. J Membrane Biol 2014; 247: 1239-51. doi:10.1007/ s00232-014-9696-2

48. Hudej R, Turel I, Kanduser M, Scancar J, Kranjc S, Sersa G, et al. The Influence of electroporation on cytotoxicity of anticancer ruthenium (III) complex KP1339 in vitro and in vivo. Anticancer Res 2010; 30: 2055-63.

49. Calvet CY, Famin D, Andre FM, Mir LM. Electrochemotherapy with bleomycin induces hallmarks of immunogenic cell death in murine colon cancer cells. Oncoimmunology 2014; 3: e28131. doi:10.4161/onci.28131

50. Cemazar M, Golzio M, Sersa G, Escoffre JM, Coer A, Vidic S, et al. Hyaluronidase and collagenase increase the transfection efficiency of gene electrotransfer in various murine tumors. Hum Gene Ther 2012; 23: 128-37. doi:10.1089/hum.2011.073

51. Bartel C, Bytzek AK, Scaffidi-Domianello YY, Grabmann G, Jakupec MA Hartinger CG, et al. Cellular accumulation and DNA interaction studies of cytotoxic trans-platinum anticancer compounds. J Biol Inorg Chem 2012; 17: 465-74. doi:10.1007/s00775-011-0869-5

52. Khazanov E, Barenholz Y, Gibson D, Najajreh Y. Novel apoptosis-inducing trans-platinum piperidine derivatives: synthesis and biological characterization. J Med Chem 2002; 45: 5196-204.

53. Halamikova A, Heringova P, Kasparkova J, Intini FP, Natile G, Nemirovski A, et al. Cytotoxicity, mutagenicity, cellular uptake, DNA and glutathione interactions of lipophilic trans-platinum complexes tethered to 1-adamantylamine. J Inorg Biochem 2008; 102: 1077-89. doi:10.1016/j.jinorgbio.2007.12.015

54. Chowdhury MA, Huq F, Abdullah A, Beale P, Fisher K. Synthesis, characterization and binding with DNA of four planaramineplatinum (II) complexes of the forms: trans- $\mathrm{PtL}_{2} \mathrm{Cl}_{2}$ and $\left[\mathrm{PtL}_{3} \mathrm{Cl}\right] \mathrm{Cl}$, where $\mathrm{L}=3$-hydroxypyridine, 4-hydroxypyridine and imidazo(1,2-alpha)pyridine. J Inorg Biochem 2005; 99: 1098-112.

55. Novakova O, Kasparkova J, Malina J, Natile G, Brabec V. DNA-protein crosslinking by trans- $\left[\mathrm{PtCl}_{2}(\mathrm{E} \text {-iminoether })_{2}\right]$. A concept for activation of the trans geometry in platinum antitumor complexes. Nucleic Acids Res 2003; 31: 6450-60.

56. Richards AD, Rodger A. Synthetic metallomolecules as agents for the contro of DNA structure. Chem Soc Rev 2007; 36: 471-83.

57. Martincic A, Cemazar M, Sersa G, Kovac V, Milacic R, Scancar J. A nove method for speciation of Pt in human serum incubated with cisplatin, oxaliplatin and carboplatin by conjoint liquid chromatography on monolithic disks with UV and ICP-MS detection. Talanta 2013; 116: 141-8. doi:10.1016/j.talanta.2013.05.01

58. Martincic A, Milacic R, Cemazar M, Sersa G, Scancar J. The use of CIM-DEAE monolithic chromatography coupled to ICP-MS to study the distribution of cisplatin in human serum. Anal Methods 2012; 4: 780-90. doi:10.1016/j. chroma.2014.10.054 